\title{
iPSC-derived functional human neuromuscular junctions model the pathophysiology of neuromuscular diseases
}

\author{
Chuang-Yu Lin, ${ }^{1}$ Michiko Yoshida, ${ }^{1,2}$ Li-Tzu Li, ${ }^{3}$ Akihiro Ikenaka, ${ }^{1}$ Shiori Oshima, ${ }^{4}$ \\ Kazuhiro Nakagawa, ${ }^{4}$ Hidetoshi Sakurai, ${ }^{1}$ Eriko Matsui, ${ }^{4}$ Tatsutoshi Nakahata, ${ }^{1}$ \\ and Megumu K. Saito ${ }^{1}$ \\ 'Department of Clinical Application, Center for iPS Cell Research and Application (CiRA), Kyoto University, Kyoto, Japan. \\ ${ }^{2}$ Department of Pediatrics, Kyoto Prefectural University of Medicine, Kyoto, Japan. ${ }^{3}$ Institute of Atomic and Molecular \\ Sciences, Academia Sinica, Taipei, Taiwan. ${ }^{4}$ Sony Imaging Products \& Solutions Inc., Tokyo, Japan.
}

The control of voluntary skeletal muscle contraction relies on action potentials, which send signals from the motor neuron through the neuromuscular junction (NMJ). Although dysfunction of the NMJ causes various neuromuscular diseases, a reliable in vitro system for disease modeling is currently unavailable. Here, we present a potentially novel 2-step, self-organizing approach for generating in vitro human NMJs from human induced pluripotent stem cells. Our simple and robust approach results in a complex NMJ structure that includes functional connectivity, recapitulating in vivo synapse formation. We used these in vitro NMJs to model the pathological features of spinal muscular atrophy, revealing the developmental and functional defects of NMJ formation and NMJ-dependent muscular contraction. Our differentiation system is therefore useful for investigating and understanding the physiology and pathology of human NMJs.

Conflict of interest: SO, KN, and EM are employed by Sony Imaging Products \& Solutions, Inc.

Copyright: (c) 2019, American Society for Clinical Investigation.

Submitted: August 31, 2018 Accepted: August 8, 2019 Published: September 19, 2019.

Reference information: JCI Insight 2019;4(18):e124299. https://doi.org/10.1172/jci. insight.124299.

\section{Introduction}

The neuromuscular junction (NMJ) is a specialized synapse that transmits signals from motor neurons (MNs) to skeletal muscle (1). Its formation is a multistep process that requires coordinated signals from MNs, skeletal muscle, glial cells, and the extracellular matrix $(2,3)$. There is growing evidence that maintaining and altering the development of NMJs or the skeletal muscle itself contribute to the pathology of NMJ-related diseases (4-6). However, the precise mechanisms of human NMJ (hNMJ) formation and pathophysiology are largely unknown, in part due to insufficient in vitro models. Several attempts have been made to reconstruct in vitro NMJs. For example, coculturing human induced pluripotent stem cell-derived (hiPSC-derived) MNs and primary muscle cells or cell lines was found to cluster acetylcholine receptors (AChRs) (6-9). Although the functional connectivity of these cells proves the ability of hiPSC-derived MNs to form functional NMJs (9), whether the morphological maturation of in vitro hNMJs corresponds to in vivo NMJs has not been elucidated. Furthermore, a mature and functional model of hNMJs composed of pluripotent stem cell-derived neurons and muscles is desired for dissecting the precise mechanisms of hNMJ pathologies. hiPSC-derived MNs and myotubes have also been shown to form NMJ-like connections $(10,11)$, but again the morphological integrity and functional maturation await further investigation.

In the current study, we successfully established a potentially novel strategy for generating in vitro hNMJs by temporarily expressing myogenic differentiation 1 (MYOD1) in hiPSCs. This structure recapitulated early developmental processes, and after 3 weeks of differentiation we could obtain a complex tissue that contained neurons, myotubes, and Schwann cells and exhibited NMJ function upon optogenetic stimulation. Using this approach, we also modeled spinal muscular atrophy (SMA) with isogenic hiPSCs by knocking down the survival of motor neuron (SMN) protein expression. The knockdown caused degenerated features in the NMJ and muscle, and the NMJ showed functional defects consistent with SMA when stimulated chemically or optogenetically. We propose our hNMJ model for investigating the physiology and pathology of hNMJs and for evaluating related therapeutic molecules. 


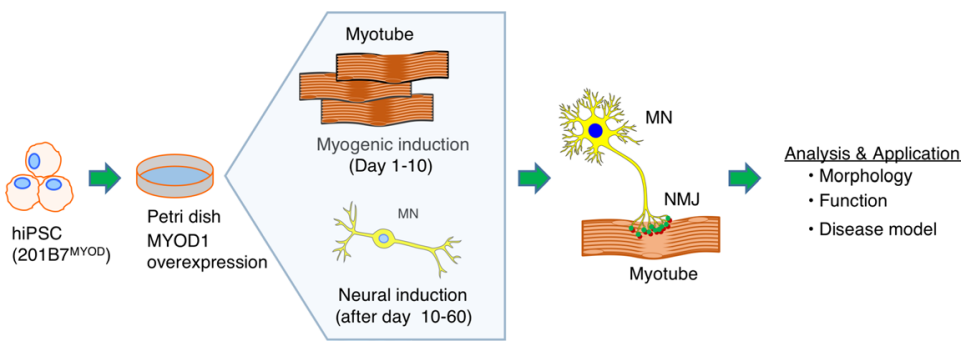

Figure 1. Schematic illustration of in vitro hNMJ formation. An illustration of an NMJ formation in a single well.

\section{Results}

Formation of hNMJs from iPSCs in a single well. The process of NMJ formation from hiPSCs in this study is illustrated in Figure 1. The transient expression of MYOD1 by doxycyclineDox-inducible (Dox-inducible) vectors converts hiPSCs efficiently to mature myotubes (12). We first established the 201B7-iPSC clone with Dox-inducible MYOD1 and mCherry expression vector (Supplemental Figure 1A; supplemental material available online with this article; https://doi.org/10.1172/jci.insight.124299DS1) (hereafter referred to as 201B7 ${ }^{\mathrm{MYOD}}$ ). By administering Dox, 201B7 ${ }^{\mathrm{MYOD}}$ differentiates into myotubes depending on the expression of exogenous MYOD1 (Supplemental Figure 1D). Upon switching the culture medium to one suitable for neuronal differentiation and maturation (13) at day 10 (Figure 2A), we detected both myosin heavy chainpositive $\left(\mathrm{MYH}^{+}\right)$myotubes and Tuj1 ${ }^{+}$neurons (Figure $\left.2 \mathrm{~B}\right)$. We also detected AChRs clustering on the surface of myotubes and juxtaposed to the axon terminal on day 21 and day 60, which indicates the emergence of NMJ-like structures (Figure 2B). The clustering of synaptic nuclei and the formation of end plate structures were also observed (Supplemental Figure 1B and ref. 14). Distribution of neuronal and skeletal muscle markers (neurofilaments, SV2, and AChRs) indicated NMJ-like patterns (Figure 2C and Supplemental Figure 1C). Upregulated expressions of genes associated with pre- and postsynaptic NMJ cell types, such as skeletal muscles (myogenin; Actin, alpha skeletal muscle [ACTA1]; and dystrophin) and MNs (SRY-box transcription factor 1 [Sox1] and Homeobox HB9 [HB9]), were also detected (Supplemental Figure 1, E-I). Immunostaining confirmed the expression of myocyte-specific enhancer factor $2 \mathrm{C}(\mathrm{MEF} 2 \mathrm{C})$ and MYH for myoblasts and HB9, Islet1, and choline acetyltransferase (ChAT) for MNs (Figure 3, A and B). The Schwann cell marker S100 was also detected in the culture (Figure 3C and Supplemental Figure 1J). To compare the effect of the culture condition on NMJ formation and original myogenic induction (12), the NMJ frequency was quantified from different culture conditions at day 30 (see Image quantification in Methods for the definition of frequency). The areas of the neurons and AChRs in the NMJ condition were significantly higher than in the myogenic condition (Supplemental Figure 2). Scanning electron microscopy images revealed swelling axon terminals binding to the myotubes (Figure 4A). Synaptic boutons capped by Schwann cells and myelinated axons integrated with myotubes in NMJ differentiation culture were confirmed by transmission electron microscopy (TEM) (Figure 4B). Synaptic vesicles and mitochondria accumulated at the pre- and postsynaptic sites of NMJs and were clearly separated by electron-dense active zones (Figure 4, C and D). These observations suggest that our in vitro NMJs expressed major cell types and promising architecture.

Interactions between myotubes and neurons are important for their differentiation. Interactions among different cell types are prerequisites for developing complicated tissue structures in vivo. In in vitro models, coculturing pluripotent stem cell-derived sympathetic neurons with primary cardiomyocytes is a critical step for neuron maturation (15). We therefore wondered whether interactions between myotubes and neurons are important for their differentiation. We first sorted $\mathrm{mCherry}^{+}$cells to address whether MYOD1-expressing cells could differentiate into neurons (Supplemental Figure 3A). Even after sorting $\mathrm{mCherry}^{+}$cells, Tuj1 ${ }^{+}$neurons emerged, although the frequency was less than in the unsorted sample (Supplemental Figure 3, B and C). These results suggest both MYOD1-expressing cells and non-MYOD1-expressing cells differentiate into neuronal cells. To gain insight into the condition that initiates NMJ formation, we cocultured green fluorescent proteintagged (GFP-tagged) iPSCs without the MYOD1 expression vector $\left(201 \mathrm{~B} 7^{\mathrm{GFP}}\right.$ ) with $201 \mathrm{~B} 7^{\mathrm{MYOD}}$ cells (Supplemental Figure 4A). Interestingly, some 201B7GFP cells differentiated into $\mathrm{MYH}^{+}$myotubes in the coculture (Supplemental Figure 4B). Conditioned medium collected from the myogenic differentiation culture only rarely induced the myogenic differentiation of $201 \mathrm{~B} 7^{\mathrm{GFP}}$, indicating that cell-cell contacts are required for 

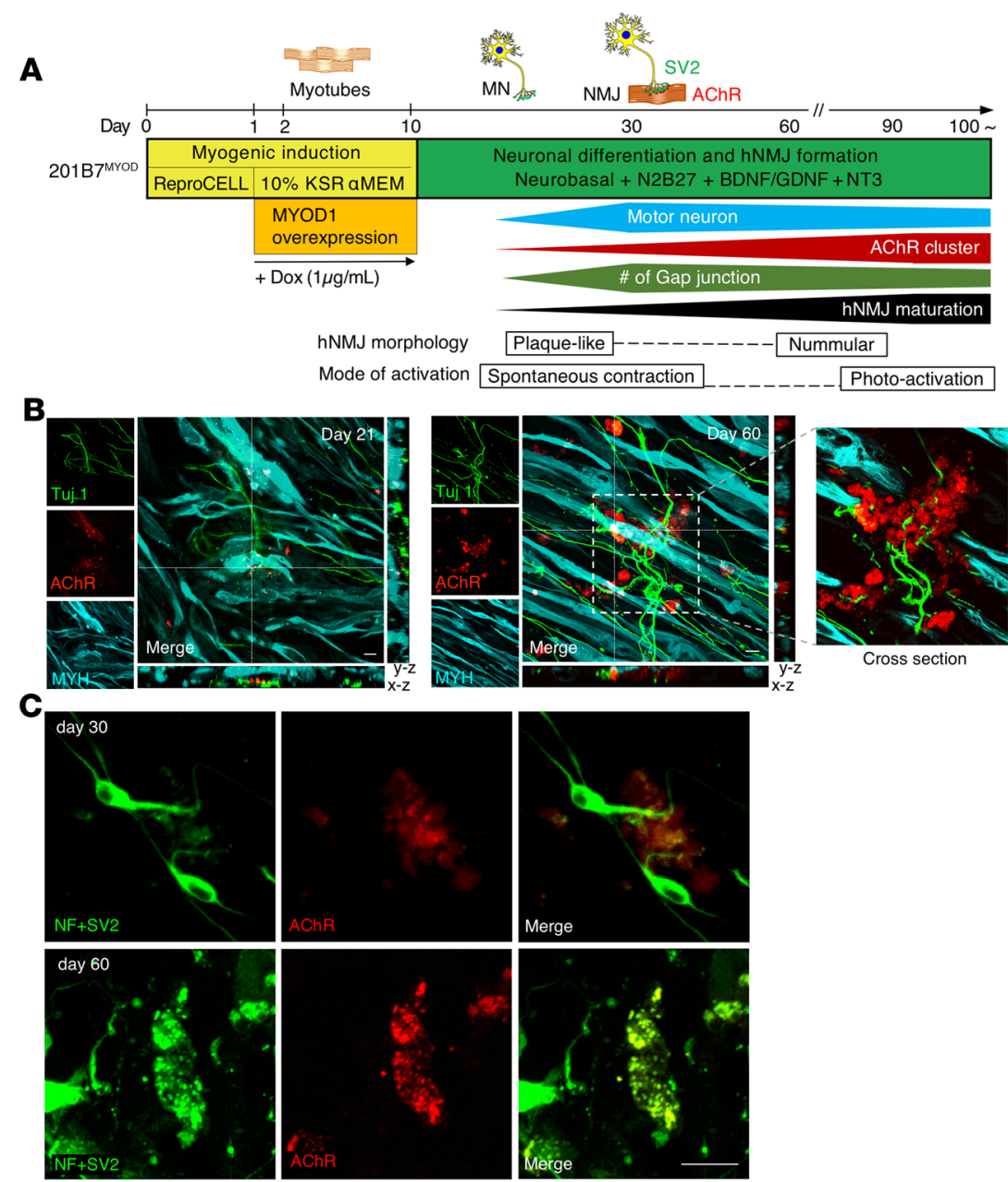

Figure 2. In vitro hNMJ formation and morphology. (A) The induction steps for the in vitro hNMJ formation from hiPSCs and temporal events in the culture system. SV2, synaptic vesicle protein 2; BDNF/GDNF, brain-derived neurotrophic factor/glial cell-derived neurotrophic factor; NT3, neurotrophin-3. (B) Morphologies of pre- and postsynaptic features at day 21 and day 60 with $z$ axis views. Tuj1-labeled neurons in the presynaptic phase. AChR is labeled by a-BTX-647. MYH, skeletal muscle marker. (C) The morphology of AChR clustering and an NMJ at day 30 and day 60 . NF, neurofilaments. See also Supplemental Figure 1, B and C. Scale bars: $10 \mu \mathrm{m}$ (B and C).

the myogenic differentiation (Supplemental Figure 4, C and D). Interestingly, conditioned medium from the 201B $7^{\mathrm{MYOD}}$ myogenic differentiation culture could produce a comparable number of Tuj $1^{+}$neurons from $201 \mathrm{~B} 7^{\mathrm{GFP}}$, indicating that a diffusible component, such as a neurotrophic factor, secreted during muscle differentiation facilitates neuronal differentiation and survival (Supplemental Figure 4, C and D). Collectively, the coexistence of myotubes and neurons facilitates the differentiation of both cell types, which is important for the development of in vitro NMJs.

hNMJ follows developmental steps in vivo. We then observed whether our hNMJ culture recapitulated the structural and morphological changes of in vivo synapse formation (16-19). The clustering on day 21 shown in Figure 2B was "plaque like," suggesting immature NMJs. By day 60, the plaques perforated and perfectly aligned with $\mathrm{MN}$ axon terminals, giving the junction a nummular (coin-shaped patches) appearance. Nummular morphology is a unique morphology of hNMJs that distinguishes them from murine NMJ morphology (20). In parallel with the AChR morphological change, the AChR subunit shifted from $\gamma(\mathrm{AChR} \gamma)$ to $\varepsilon(\mathrm{AChR} \varepsilon)$ (Supplemental Figure 5), which is an important hallmark of adult-type AChRs (21). In the development of NMJs in vivo, multiple innervations occur at first, but all but 1 axonal input to each myotube will be removed as a result of neuronal pruning (22-24). In our culture condition, the area of neurons did not increase in long-term culture, but the area of AChRs and NMJs increased significantly at the later stage (Supplemental Figure 6). The ratio of Islet1 ${ }^{+} \mathrm{MNs}$ among neuronal nuclei ${ }^{+}\left(\mathrm{NeuN}^{+}\right)$ 
A
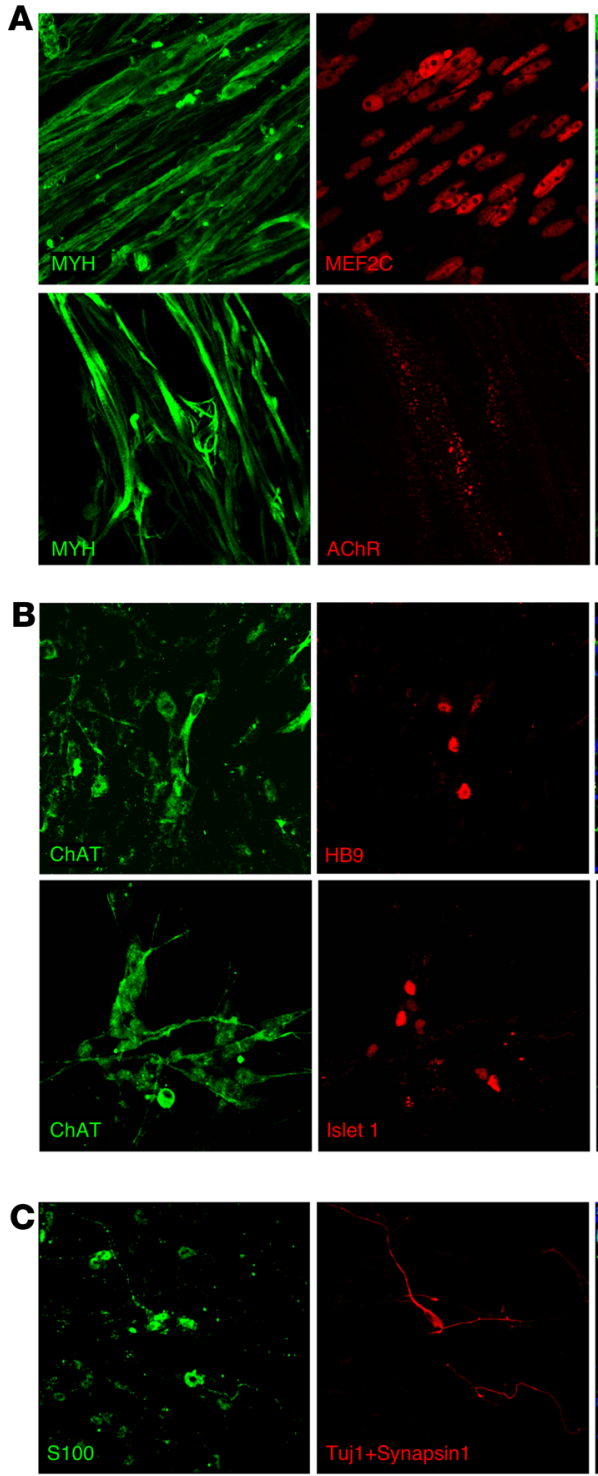
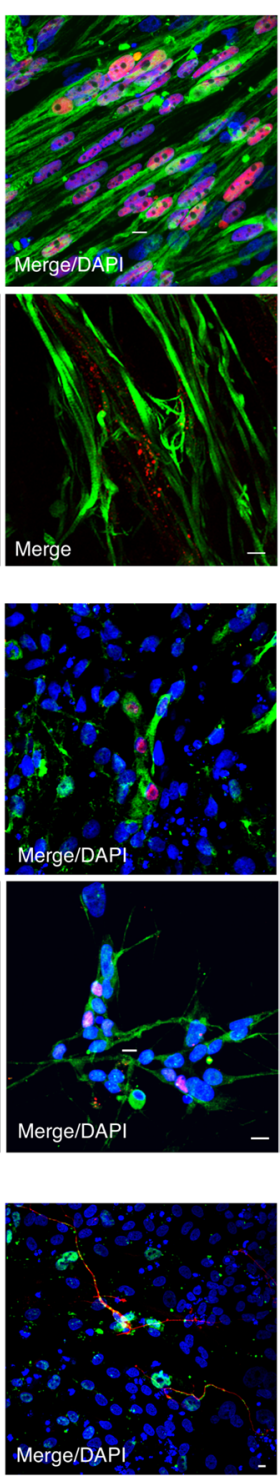

Figure 3. Pre- and postsynaptic components of in vitro NMJ. (A) Expression of the myogenic markers MYH, MEF2C, and AChR. (B) Expression of the MN-specific markers HB9 Islet1, and choline acetyltransferase (ChAT) at day 30. (C) Expression of the Schwann cell marker S100 (green). Scale bars: $10 \mu \mathrm{m}$ (A-C).

neurons was $27.4 \%$ at day 60 (Supplemental Figure 7A). Flow cytometry analysis showed the percentage of $\mathrm{MNs}$ dropped with time, as $9.75 \%$ were $\mathrm{HB}^{+}$and $13.15 \%$ were Islet $1^{+}$cells in day $30 \mathrm{NMJ}$ culture and $4.17 \%$ were $\mathrm{HB}^{+}$and $11.1 \%$ were Islet $1^{+}$cells in day $60 \mathrm{NMJ}$ culture (Supplemental Figure 7B). These results indicated that neuronal selection might occur with the maturation of in vitro hNMJs. TEM imaging revealed muscle structural fibers, $\mathrm{Z}$ lines, multiply branched axon terminals, and necrosis showing synapse elimination (Figure 4, E-G). The axon terminals in the matured NMJs had synaptic vesicles clustered inside and showed cytomatrix depositions and active zones juxtaposed to the postsynaptic density (Figure 4, H and I). The protruding space of the subneural clefts was surrounded by the sarcolemma, which is where AChRs were located (Figure 4J). Overall, we observed a series of hNMJ developmental stages in our NMJ culture system.

Agrin maintains AChR clustering and hNMJ culture. We next investigated the contribution of the neuronal factor agrin in our in vitro NMJ system because agrin accumulates and stabilizes AChRs at the synaptic sites $(16,25,26)$. Agrin administration had no significant effect on the ratio of NMJs in longterm culture or vigorous myotubes and junctional folds (Figure $4, \mathrm{~K}$ and L, and Supplemental Figure 8). On the other hand, in the presence of antiagrin antibody, the area of NMJs decreased, and only remnants of myotubes and deteriorated axon terminals were found in the culture (Figure 4, K and L, and Supplemental Figure 8). These observations indicate agrin plays an important role in the formation and maintenance of our in vitro hNMJs, which mimics the role of agrin in vivo. 
A

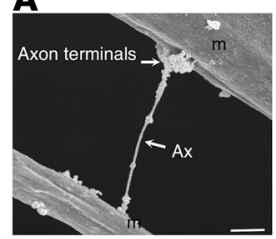

C

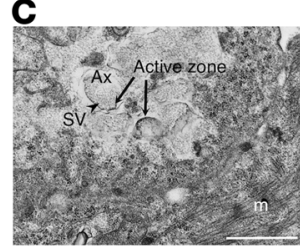

E

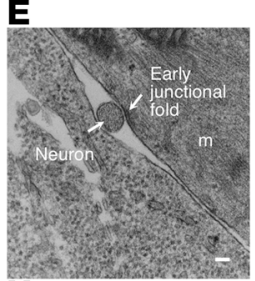

H

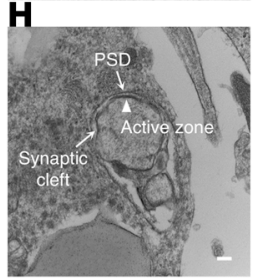

F

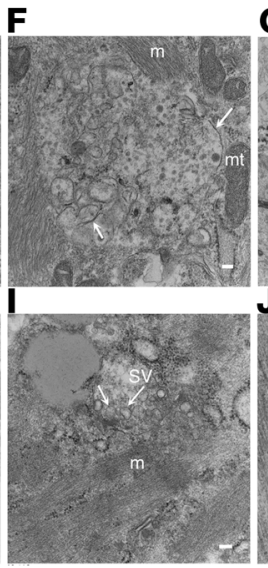

B

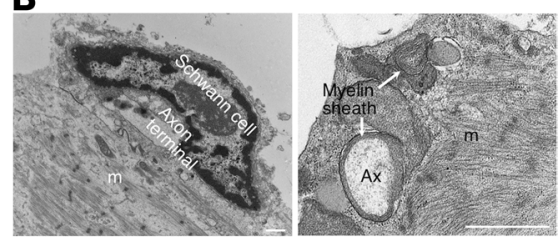

D

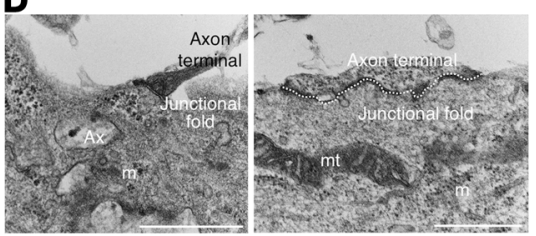

G
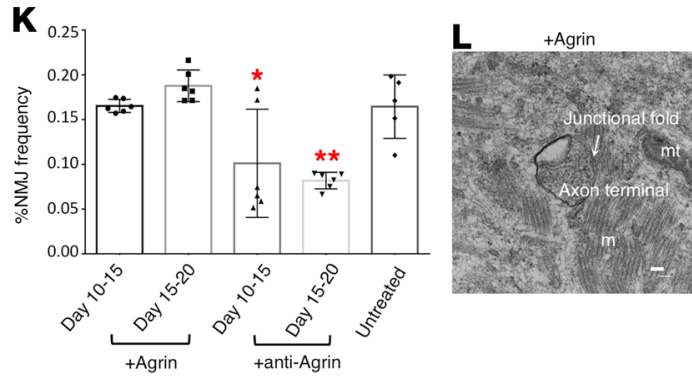

+ anti-Agrin-Ab

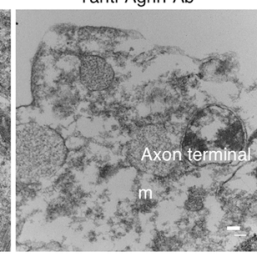

Figure 4. Fine structures of hNMJs and developmental stage analysis. (A) Scanning electron microscopy images show swelling axon terminals binding to a myotube. (B) TEM images show a synaptic bouton capped by the terminal Schwann cell and myelinated axon. (C) The TEM image shows synaptic vesicles (arrowheads) in presynaptic sites, active zones (arrows), and mitochondria accumulated in postsynaptic sites. (D) Enlarged axon terminals observed inside a myotube. Junctional folds are seen as electron-dense areas. (E) A neuron innervating a myotube $(\mathrm{m})$ and an electron-dense area observed on the muscle plasma membrane. (F) Multiply branched axon terminals observed in a myotube. Electron-dense areas (arrows) locate at each axon terminal. Mitochondria (mt) are found nearby. (C) Necrosis was observed among the axon terminals (arrows). (H) An active zone (arrowhead) juxtaposed to the postsynaptic density (PSD, arrow) with a synaptic cleft observed in between. (I) Synaptic vesicles (SV) docked to the active zone (arrows). (J) A subneural cleft surrounded by sarcolemma with AChRs residing inside. (K) NMJ frequencies with agrin and antiagrin treatment in NMJ cultures at day 30. See also Supplemental Figure 8. (L) Change in NMJ morphology with agrin or antiagrin antibody treatment. Scale bars: $1 \mu \mathrm{m}(\mathbf{A}-\mathbf{D}), 100 \mathrm{~nm}(\mathbf{E}-\mathbf{J}$ and $\mathbf{L})$. (K) ${ }^{*} P<0.05$; ${ }^{*} P<0.01$ (1-way ANOVA with Tukey's test relative to untreated control). Data are shown as mean $\pm S E M ; n=6$ (agrin and antiagrin); $n=5$ (untreated control). Each dot represents a biologically independent sample.

AChR signal-dependent spontaneous synchronized contraction of skeletal muscle in early hNMJ culture. During days 15-30, the entire area of each culture showed spontaneous, synchronized movement that resembled contractions. Furthermore, the movement synchronized with the intracellular $\mathrm{Ca}^{2+}$ concentration of the myotubes (Figure 5, A and B) and was inhibited by the skeletal muscle relaxant dantrolene, confirming that the movement was myotube contractions (Supplemental Figure 9A and ref. 27). Muscle excitation occurred robustly, as we could observe $\mathrm{Ca}^{2+}$ oscillations in all wells in a 96-well plate (Supplemental Figure 9, B and C). 

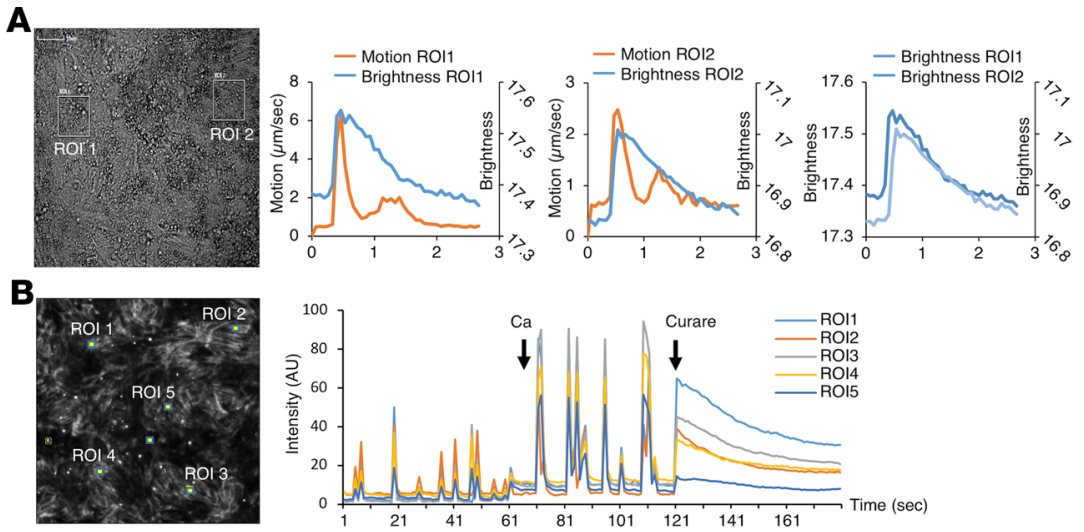

C
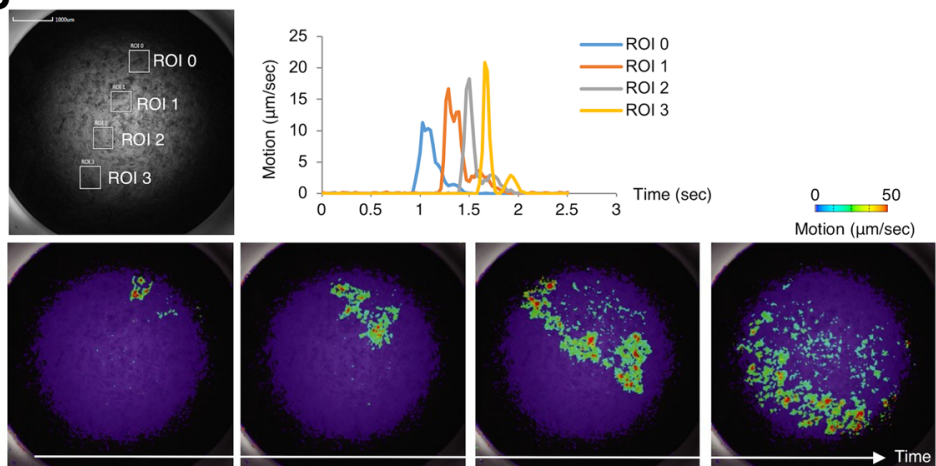

D
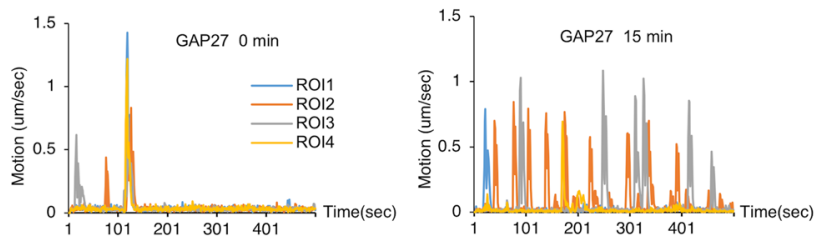

Figure 5. Functional analysis of in vitro hNMJ culture at day $\mathbf{2 0}$ of NMJ differentiation. (A) $\mathrm{Ca}^{2+}$ transients and movements of the myotube were measured simultaneously in 2 regions of interest (ROIs). (B) Ca ${ }^{2+}$ imaging in $5 \mathrm{ROIs}^{2} \mathrm{Ca}^{2+}$ transients increased with the application of calcium and stopped with the application of curare. (C) Motion analysis in 4 ROls. (D) Motion analysis of myotubes before and after treatment with a gap junction inhibitor, GAP27. GAP27 was not washed out in the experiment. See also Supplemental Figure 9 and Supplemental Videos 1 and 2.

The excitation of myotubes could be diminished by curare, a competitive inhibitor of AChRs (28), indicating that the $\mathrm{Ca}^{2+}$ oscillations were mediated by AChR signaling (Figure 5B and Supplemental Video 1). Therefore, the muscle excitation was not spontaneous but governed by spontaneous neuronal activity via functional synaptic connections between the neurons and the myotubes. During the development of NMJs, gap junctions, which are intercellular channels between myotubes that enable the cell-cell propagation of electrical activity, are transiently expressed $(29,30)$. By increasing the temporal resolution of the motion vector analysis, we found the contractions were not completely synchronized but propagated to adjacent regions (Figure 5C and Supplemental Video 2). The propagation was disrupted by a gap junction inhibitor, GAP27, indicating action potentials were propagated through gap junctions (Figure 5D). The temporal expression of connexin 43 and the electrical integration observed in our hNMJ system was compatible with that of in vivo NMJ development (Supplemental Figure 9, D-G). In aggregation, these functional analyses indicate that in vitro NMJs at this phase (days 15-30) recapitulate key features of early in vivo NMJ development.

Optogenetic neuronal excitation controls muscular contraction in mature hNMJ culture. In adult NMJs in vivo, spontaneous muscle contractions usually do not occur because of the loss of spontaneous neuronal excitation and gap junction-dependent electrical connections in myotubes (31). Although in vitro tracking of hNMJ development is an advantage of our system, it also causes the spontaneous halting of muscular contraction and limits the period of functional evaluation. To overcome these issues and to confirm the function 
of mature in vitro NMJs, we activated neurons by introducing a photoactivating channel into the NMJ culture by lentivirus infection (Figure 6A). To avoid any interference of the spontaneous contractions at the early stage of the NMJ culture, we used long-term NMJ culture (beyond day 90) for the photostimulation study. The engineered neuronal cells were visualized by the expression of channelrhodopsin-enhanced yellow fluorescent protein (channelrhodopsin-EYFP) driven by synapsin 1 promoter at day 100 of the NMJ differentiation (Figure 6A). Muscle contraction was triggered only by blue light, which confirmed the photoactivated system was established (Figure 6B and Supplemental Video 3). No contraction was observed in the well without ChR2-EYFP even when the cells were stimulated with blue light. Contractions were completely inhibited by curare administration in all cultures ( 3 biologically independent cultures were tested), confirming muscle contractions were caused by the ACh/AChR route (Figure 6C and Supplemental Video 4). Immunostaining of the samples used for the optogenetic experiments confirmed the formation of NMJs (Supplemental Figure 10A). Exposure to pulsed blue light at various frequencies also triggered synchronous muscle contraction (Supplemental Figure 10, B-E, and Supplemental Videos 5-8). Overall, the optogenetic NMJ system supported the functional maturation of in vitro NMJs in long-term culture.

Modeling the pathophysiology of SMA with our hNMJ system. To validate the application of our hNMJ system, we modeled the pathophysiology of SMA, a congenital neuromuscular disease caused by a decreased expression of the SMN protein. Classically, SMA is regarded as a motor neuronal disease, but recent animal and hybrid models have indicated that the earliest morphological defects occur at the NMJ $(32,33)$, indicating SMA is an NMJ disease. Because a human model to study NMJ function is lacking, we knocked down SMN mRNA by shRNA in 201B7MYOD $\left(201\right.$ B $^{\text {MYOD }}-\mathrm{SMN}^{\mathrm{KD}}$ ) to study the molecular pathology of SMA on an isogenic background $(34,35) .201 \mathrm{~B}^{\mathrm{MYOD}}-\mathrm{SMN}^{\mathrm{KD}}$ iPSCs showed a significant decrease in mRNA level and a 60\% decrease in SMN protein expression (Supplemental Figure 10, F and G). Neurons and clustered AChRs were detected in NMJ culture of 201B7 ${ }^{\mathrm{MYOD}}{ }_{-S M N}{ }^{\mathrm{KD}}$ iPSCs at day 30 . The area of the NMJ in 201B7 ${ }^{\text {MYOD }}-S M N^{K D}$ was significantly smaller than in 201B7MYOD (Figure 7A). Scanning electron microscopy images revealed bunches of myotubes and axons that were well extended and anchored to the myotubes with enlarged axon terminals in control 201B7 ${ }^{\mathrm{MYOD}}$ culture but not in 201B7 ${ }^{\mathrm{MYOD}}-\mathrm{SMN}^{\mathrm{KD}}$ culture (Figure 7B). Subcellular details of the myotubes and NMJ-related structures of 201B7 ${ }^{\mathrm{MYOD}_{-} \mathrm{SMN}} \mathrm{ND}^{\mathrm{KD}}$ were also distinct from 201B7 ${ }^{\mathrm{MYOD}}$ (Figure 7C). The mitochondria morphology and integrity were damaged in 201B7 ${ }^{\mathrm{MYOD}}-\mathrm{SMN}{ }^{\mathrm{KD}}$, and myofibers had less integrity in their structure. These results are consistent with recent findings of decreased mitochondrial biogenesis and pathological changes in the muscle of patients with SMA (36). To evaluate functional variations, we next observed the amplitude and patterns of NMJ-dependent muscle contractions. 201B7 ${ }^{\mathrm{MYOD}}-\mathrm{SMN}^{\mathrm{KD}}$ showed a smaller area of contractions than control 201B7MYOD (Figure 7D and Supplemental Videos 9 and 10). Furthermore, the synchronicity of contraction was disrupted, and the maximum speed of muscular contraction was decreased (Figure 7D and Supplemental Videos 9 and 10). Intriguingly, in 201B7 ${ }^{\mathrm{MYOD}}-\mathrm{SMN}^{\mathrm{KD}}$, myotubes were often observed to break during muscular contraction, a fragility not found in 201B7 ${ }^{\mathrm{MYOD}}$ (Supplemental Figure 10H). We also applied optogenetics to $201 \mathrm{~B}^{\mathrm{MYOD}}-\mathrm{SMN}^{\mathrm{KD}}$ but could not detect muscle contraction (Figure $7 \mathrm{E}$ and Supplemental Video 11), indicating the loss of NMJ function in SMA at the later stage. These phenomena implied the potential susceptibility of SMA myotubes to muscle contraction, which may be associated with the muscle atrophy seen in patients with SMA. Overall, the suppressed expression of SMN during NMJ development resulted in decreased AChR clustering with functional deficit and impaired muscular viability. To conclude, our hNMJ system can be applied to recapitulate the in vivo phenotypes of human neuromuscular diseases, such as SMA, allowing us to evaluate the function of NMJ in vitro.

hNMJ features recapitulated with human embryonic stem cells. To substantiate the reproducibility of our hNMJ culture established with 201B ${ }^{\mathrm{MYOD}}$, we evaluated hNMJ formation with another human pluripotent stem cell clone. For this, we introduced the Dox-inducible MYOD1 expression vector into human embryonic stem cell (hESC) line H9 (hereafter referred to as H9MYOD). When H9MYOD was differentiated, the neurons and clusters of AChRs were observed at day 30 and day 60 (Supplemental Figure 11A). TEM imaging revealed features of a mature $\mathrm{NMJ}$, including muscle fibers, axon terminals with synaptic vesicles clustered inside, cytomatrix depositions, and active zones juxtaposed to the postsynaptic density (Supplemental Figure 11B). Through calcium imaging assays and motion analysis, we observed calcium sparks and muscle contractions were triggered by $\mathrm{Ca}^{2+}$. Further, the contractions could be interrupted with curare treatment (Supplemental Figure 11C and Supplemental Videos 12 and 13). These results collectively indicate that our NMJ differentiation strategy is applicable to multiple cell lines. 
A
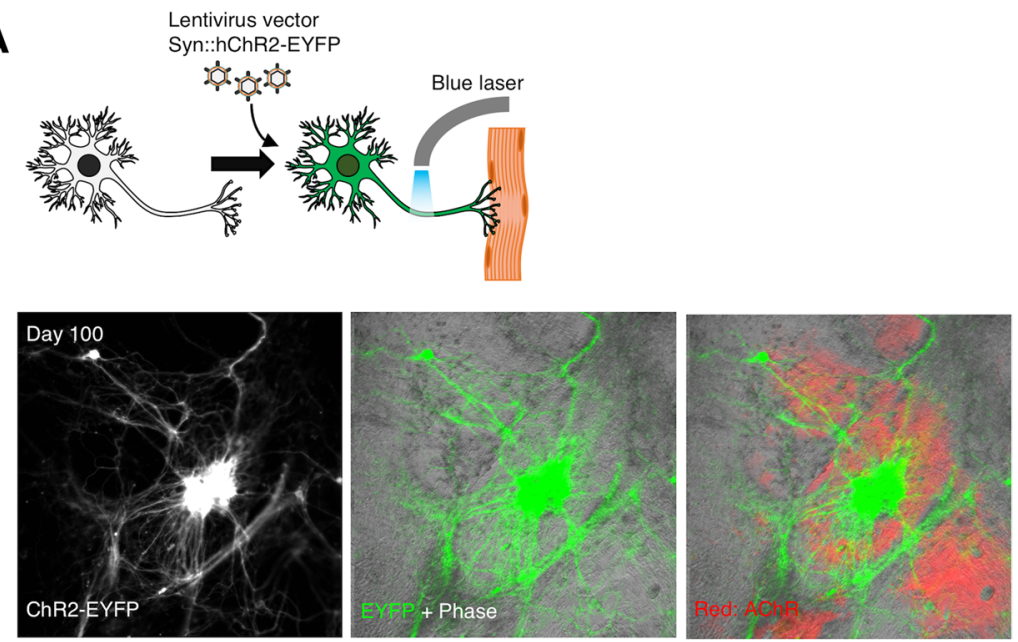

B B: Ex 488nm R: Ex $647 \mathrm{~nm} *$ All images in the same FOV

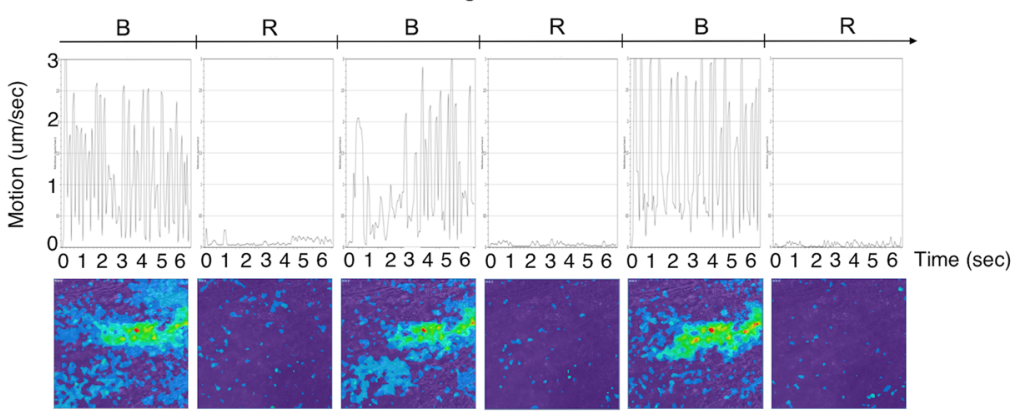

C
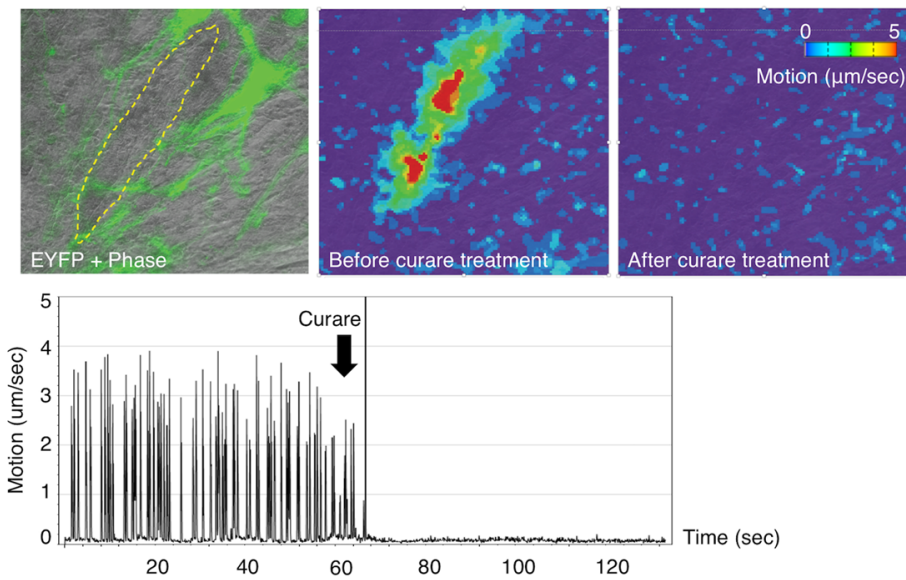

Figure 6. Motion analysis of photoactivated in vitro hNMJ culture. (A) Neurons expressing ChR2-EYFP (green) and AChR (red) in hNMJ culture at day 100. (B and C) Motion analysis of the culture. (B) Muscle contraction was triggered only by blue light $(488 \mathrm{~nm})$. Color bar is shown in C. (C) The contraction was inhibited by curare. The yellow dotted area encloses the muscle, and the green indicates neurons at the left. See also Supplemental Videos 3 and 4.

\section{Discussion}

The present study is the first to our knowledge to generate in vitro hNMJs without coculturing 2 types of cells in a single well. Previous studies generated NMJs by coculturing MNs and skeletal muscle cells derived from hESCs, iPSCs, or primary cultures $(8,11,37)$. Protocols for coculturing require complex media combinations and time-consuming induction steps that last several months. Even then, the MNs and muscle are developmentally immature and have short life spans and low cell efficiency (38). Our simple and robust approach results in a mature NMJ structure that includes functional connectivity, recapitulating in vivo synapse formation. Although more research is required for understanding the underlying biology of in vitro NMJs, our study provides a promising framework for iPSC-based development and pathology of the NMJ. 


\section{A}
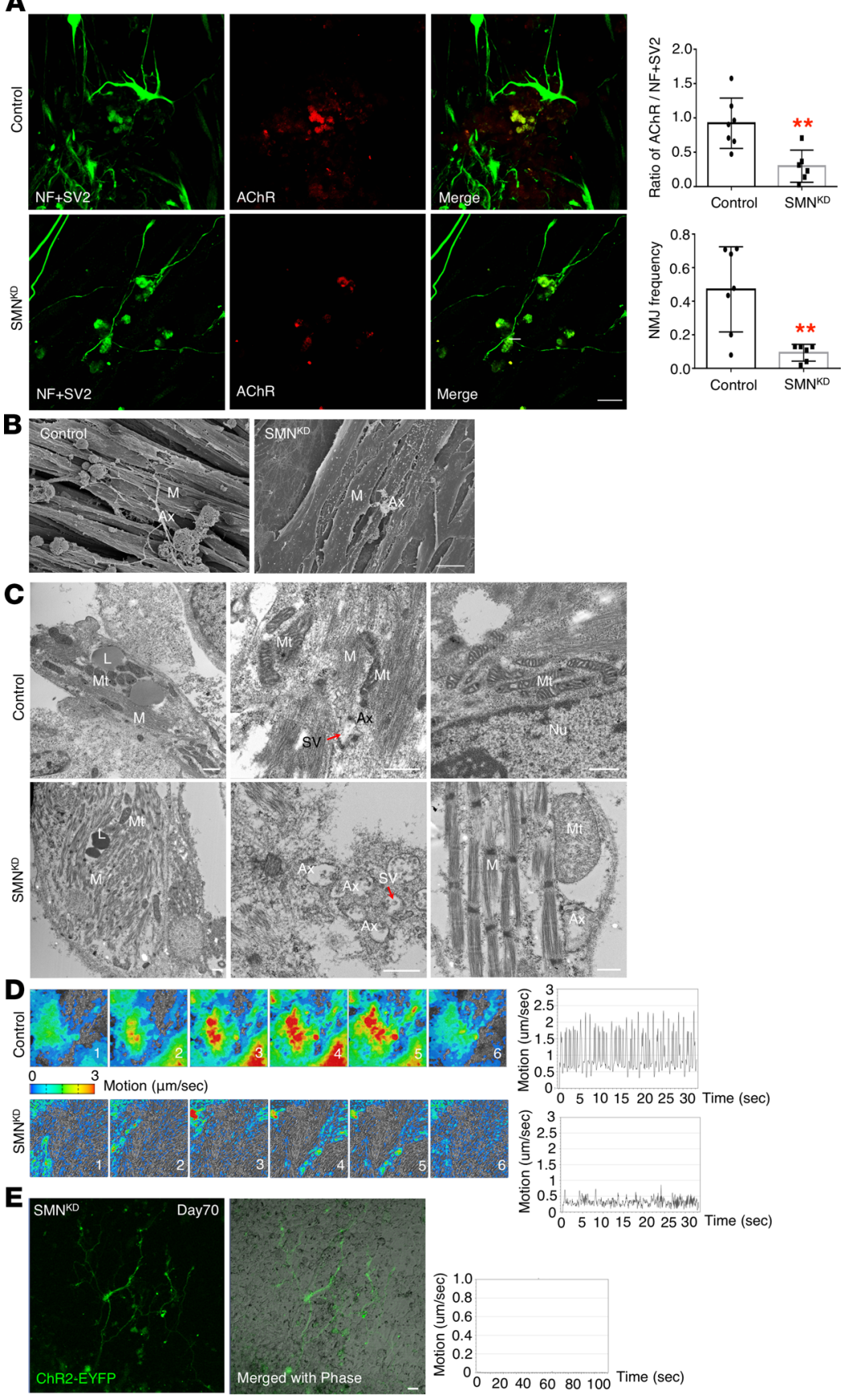

Figure 7. hNMJ differentiated from 201B7 ${ }^{M Y 0 D}-S M N^{K D}$ iPSCs at day 30. (A) The morphology of AChR clustering and NMJs from $201 \mathrm{~B}^{\mathrm{MYOD}}$ (control) and $201 \mathrm{~B}^{\mathrm{MYOD}}-\mathrm{SMN}^{\mathrm{KD}}\left(\mathrm{SMN}{ }^{\mathrm{KD}}\right.$ ) iPSCs at day 30. ${ }^{* *} P<0.01$ (unpaired 2-sided Student's $t$ test). Data are shown as mean $\pm \mathrm{SEM} ; n=6$. Each dot represents a biologically independent sample. (B) Scanning electron microscopy images show the morphology of myotubes and axon terminals in $201 \mathrm{~B} 7^{\mathrm{MYOD}}$ and SMN ${ }^{\mathrm{KD}}$ cultures. (C) TEM images show subcellular features of myotubes and neuron axons in 201B $7^{\mathrm{MroD}}$ and $\mathrm{SMN} \mathrm{KD}^{\mathrm{KD}}$ cultures. (D) The time-series contraction pattern of myotubes in $201 \mathrm{~B}^{\mathrm{MYOD}}$ and $201 \mathrm{~B}^{\mathrm{MYOD}}-\mathrm{SMN}{ }^{\mathrm{KD}}$ differentiation culture at day 30 . (E) Optogenetics of SMN ${ }^{K D}$ and motion analysis at day 70. See also Supplemental Figure 10, F-H, and Supplemental Videos 9-11. Scale bars: $10 \mu \mathrm{m}$ (A and B), 500 nm (C). M, myotube; L, lipid; Nu, nucleus.

The hNMJ is mainly composed of 3 types of cells: MNs, skeletal muscle, and Schwann cells, all of which are differentiated by the temporal expression of MYOD1 in iPSCs. Interestingly, we found neurons with compatible features of MNs without ventralization and/or caudalization, indicating that the microenvironment, such as signals from differentiating myogenic cells, in our culture induces the commitment of neuronal progenitors toward MNs. We hypothesized that factors involved in promoting the myogenic potential of stem 
cells are produced and secreted by myoblasts to enhance the in vitro differentiation of stem/progenitor cells into neurogenic lineage. Another possibility is that the temporal expression of MYOD converts some iPSCs into bipotential stem/progenitor cells. The existence of "bipotential axial stem cells" as precursors of both posterior neural plate cells and paraxial mesodermal cells has been reported (39). Considering that human organoids from human pluripotent stem cells usually require 3-dimensional aggregation for their formation, our culture shows a unique possibility that optimization of the 2-dimensional culture condition enables the autonomous 3-dimensional organization of organoids from human pluripotent stem cells.

The formation and maintenance of NMJs require complicated reciprocal signals between nerve, muscle, and Schwann cells. Additionally, action potential activity in presynaptic neurons and postsynaptic myotubes can profoundly influence the structure and function of synapses. Ultimately, redundant neurons and immature NMJs are selected out as a result of neuronal pruning $(18,40)$. Indeed, the decreasing ratio of MNs in NMJ culture at day 60 suggested progressive synapse elimination at maturing NMJs. In our NMJ model, muscle contractions triggered by the spontaneous firing of MNs were observed concordantly with the expression of gap junctions. In the later culture period, we observed more morphologically mature NMJs with activated synapses, synaptic vesicles, and increased numbers of AChRs. Recent studies have indicated that hNMJs have a unique morphology that is smaller and more fragmented than murine NMJs $(20,41)$. This finding suggests the necessity of in vitro hNMJs for studying NMJ development and disease modeling. Although muscle contractions did not occur spontaneously after maturation in our system, optogenetic neuronal activation controlled the contractions in an AChR-dependent manner. Coculturing optogenetic hESC-derived MNs with human primary myoblasts has been shown to form in vitro NMJs capable of muscle contraction dependent on the ACh/AChR axis, but the AChR subunits expressed are fetal $\gamma$ instead of mature $\varepsilon$ (9). On the other hand, the ability to trigger synchronous muscle contractions with various frequencies of pulsed blue light in the present study verified our in vitro hNMJs are mature and functional (42).

As a proof of concept of our system for disease modeling, we used an SMN-KD iPSC model of SMA. We did not use patient-derived iPSCs because interpretation of the data could have been difficult because of the different genomic backgrounds from the patient and control iPSCs. Since complete SMN gene knockout is not a useful model, because this condition is lethal early in embryogenesis (43), we selected SMN mRNA KD by shRNA. The characteristics of our SMN-KD iPSC clone when differentiated into hNMJs echoed previous mouse SMA models but also highlighted a functional impairment of NMJs in SMA and the fragility of skeletal muscle cells, as too did defective responses to optogenetics. Thus, our in vitro hNMJs could be applicable to investigating the reciprocal signals required for the formation and activity-dependent plasticity of synapses. Our hNMJs could also be used to evaluate therapeutic candidates for neuromuscular diseases in a high-content screening.

To conclude, we described the generation of functional hNMJs derived from hiPSCs. These hNMJs were established in a simple 2-dimensional culture without cell sorting or passaging. Because the development of our hNMJs follows in vivo developmental stages and because our NMJs showed a fully matured appearance in prolonged culture, we demonstrated they can be used to study the development of NMJ-related diseases and have potential to evaluate the effects of related pharmacological interventions.

\section{Methods}

Cell lines. Human iPSC line 201B7 was provided by Shinya Yamanaka (CiRA, Kyoto University, Kyoto, Japan) (44), and the hESC line H9 was purchased from a stem cell bank (WiCell) (45). 201B7 ${ }^{\mathrm{MYOD}}$ and H9MYOD were constructed by transfecting Dox-inducible MYOD1 expression vector into 201B7 and H9, respectively, as previously described (12). MYOD1 expression can be monitored by mCherry expression (Supplemental Figure 1D). mTeSR medium (STEMCELL Technologies) was used to maintain the iPSCs and H9. 201B7GFP was constructed by transfecting a GFP expression vector into 201B7. The passage numbers used in this study were 70-90. We checked for mycoplasma contamination every month with the MycoAlert Mycoplasma Detection Kit (LT07-318, Lonza), and all cultures were persistently negative.

NMJ differentiation. Myogenic differentiation of $201 \mathrm{~B} 7^{\mathrm{MYOD}}$ was performed as previously described (12). Briefly, 201B7MYOD cells $\left(3 \times 10^{4} /\right.$ well/96-well plate) were seeded on a Matrigel-coated Nunc cell culture plate and differentiated into NMJs by adding Dox to myogenic differentiation medium (minimum essential medium $\alpha$ [ $\alpha$-MEM] [Thermo Fisher Scientific], 10\% KSR [knockout serum replacement] [Thermo Fisher Scientific], $100 \mathrm{mM} \beta$-mercaptoethanol [MilliporeSigma], and $50 \mathrm{U} / \mathrm{ml}$ penicillin/streptomycin [Thermo Fisher Scientific]). The neuronal differentiation was induced by changing the medium to 
Neurobasal medium (Thermo Fisher Scientific) supplemented with neurotrophic factors (BDNF, GDNF, and NT3, $10 \mathrm{ng} / \mathrm{ml}$ each [R\&D Systems], and N2 and B27, 1× each [Thermo Fisher Scientific]) from day 10 . Penicillin/streptomycin $(50 \mathrm{U} / \mathrm{ml})$ was added to the medium with neurotrophic factors, and the medium was changed every 3-4 days thereafter.

Comparison of culture conditions for NMJ formation and for myogenic induction. First, $1 \times 10^{4}, 3 \times 10^{4}, 5 \times$ $10^{4}$, and $7 \times 10^{4} 201 \mathrm{~B} 7^{\mathrm{MYOD}}$ cells were seeded on a Matrigel-coated, 96-well plate. At day 10, myogenic differentiation or NMJ differentiation medium was applied. We harvested the samples at day 30 and performed immunocytochemistry labeling for NMJ detection. Images were acquired and analyzed by an In Cell Analyzer 6000 (GE Healthcare Life Sciences).

$R N A$ isolation and quantitative PCR. Total RNA extraction from the cells was performed using the RNeasy Mini kit (QIAGEN). One microgram of total RNA was used for reverse transcription with the PrimeScript RT Master Mix (TaKaRa). Real-time PCR was performed with SYBR Premix Ex Taq II (TaKa$\mathrm{Ra}$ ) in triplicate using the StepOnePlus system (Applied Biosystems). GAPDH was used as an endogenous control. The primer sets used for the quantitative PCR assay are described in Supplemental Table 1.

Flow cytometry analysis of $\mathrm{HBO}^{+}$and Islet $1^{+}$cells in NMJ culture. Cells in NMJ cultures were harvested on day 30 and day 60. After the cells were permeabilized with Triton X-100, they were labeled with antibodies against HB9 and Islet1. The labeled cells were analyzed with BD FACSAria (BD Biosciences), and the FACS results were analyzed and processed with FlowJo software (Tree Star Inc.).

Flow sorting of $m$ Cherry ${ }^{+}$cells. 201B $7^{\mathrm{MYOD}}$ cells were seeded on a Matrigel-coated plate and differentiated into NMJs by adding Dox to myogenic differentiation medium. On day 2 of the differentiation, mCherry ${ }^{+}$ cells were sorted out using BD FACSAria and FACSDiva software. The sorted cells were replated on a Matrigel-coated, 96-well plate $\left(8.8 \times 10^{4}\right.$ cells/well $)$ and cultured in myogenic differentiation medium with or without Dox. On day 20, the ratio of neurons to myotubes was quantified by immunocytochemistry.

Conditioned medium preparation and cell-to-cell contact experiments. Conditioned medium of 201B7MYOD was collected from the myogenic differentiation culture. 201B7GFP was cultured in one of the following media to induce differentiation: (a) culture medium for myogenic differentiation plus an equal amount of conditioned medium of 201B7 ${ }^{\mathrm{MYOD}}$, (b) culture medium for neural differentiation (Neurobasal medium + $\mathrm{N} 2+\mathrm{B} 27+\mathrm{B} / \mathrm{GDNF}+\mathrm{NT} 3)$, or (c) culture medium for myogenic differentiation $(10 \% \mathrm{KSR}+\mathrm{DMEM})$. On day 20, the percentage of neurons or myotubes to $\mathrm{GFP}^{+}$cells was quantified by immunocytochemistry.

Agrin and antiagrin treatment. During the NMJ differentiation, the culture was treated with agrin (5 $\mu \mathrm{g} / \mathrm{ml})(\mathrm{R} \& \mathrm{D}$ Systems) and antiagrin $(10 \mu \mathrm{g} / \mathrm{ml})(\mathrm{R} \& \mathrm{D}$ Systems) on days 10-15 and days 15-20 in a 96-well plate, respectively. The culture was harvested until day 30 for immunocytochemistry labeling. Images were acquired and analyzed with In Cell Analyzer 6000 (GE Healthcare Life Sciences). Samples were also harvested for TEM analysis.

Motion vector analysis. Phase-contrast images with a red-filtered optical source and fluorescence (Fluo-8 [AAT Bioquest]) were captured continuously at 15 fps using a color camera, XCG-5005CR (Sony Corporation). Motion quantification with the red-separated images and brightness analysis with the green-separated images in ROIs were performed using the SI8000 Cell Motion Imaging System (Sony Corporation; https:// www.sonybiotechnology.com/us/instruments/si8000-cell-motion), which provides motion vector analysis based on the block matching method (27). Both moving image capture and motion analysis were performed using the SI8000 Cell Motion Imaging System at $38 \mathrm{fps}$. To inhibit muscle contractions, $20 \mu \mathrm{M}$ dantrolene (MilliporeSigma) was added to the culture medium. In some experiments, we used extracellular $\mathrm{Ca}^{2+}$ to induce muscular contractions. These $\mathrm{Ca}^{2+}$-triggered muscle contractions could be inhibited by curare (data not shown), confirming the involvement of AChR signaling.

Calcium imaging. 201B7 ${ }^{\mathrm{MYOD}}$ cells were seeded at a density of $2.2 \times 10^{4}$ cells/well and differentiated into NMJs in a Costar 96-well black-wall/clear-bottom plate. On day 20, the growth medium was removed, and the cells were loaded with $100 \mu \mathrm{Fluo}-8$ recording medium at $37^{\circ} \mathrm{C}$ for 1 hour. The cells were washed twice with PBS and then imaged with In Cell Analyzer 2000. The fluorescence intensity was expressed as the F/F0 ratio.

Functional drug screening system analysis. 201B ${ }^{\mathrm{MYOD}}$ cells were seeded onto a Matrigel-coated, 96-well, black-wall/clear-bottom plate at a density of $2.2 \times 10^{4}$ cells/well. Half of the medium in each well was exchanged every day until the cultures started contracting. Then, the growth medium was removed, and the cells were loaded with $100 \mu 1$ Fluo- 8 recording medium at $37^{\circ} \mathrm{C}$ for 1 hour. The cells were washed twice with PBS, and differentiation medium was added to the wells. $\mathrm{Ca}^{2+}$ oscillations were monitored with the Functional Drug Screening System (FDSS) microplate reader (Hamamatsu Photonics), and data points 
were collected at 1-minute intervals and analyzed using FDSS software (Hamamatsu Photonics) for frequency and amplitude quantification.

TEM. NMJ cultures were prefixed in $2 \%$ paraformaldehyde and $2 \%$ glutaraldehyde in $0.1 \mathrm{M}$ phosphate buffer ( $\mathrm{pH} 7.4$ ) at $4^{\circ} \mathrm{C}$ overnight. Postfixation was done in $1 \%$ osmium tetroxide solution for 1 hour at room temperature. The samples were dehydrated in graded concentrations of ethanol $(30 \%, 50 \%, 70 \%$, 90\%, 95\%, and 100\%) and embedded in Epon resin. Ultrathin sections (80 nm) were cut and stained with uranyl acetate and alkaline lead citrate (46). The specimens were examined with a transmission electron microscope (H-7650, Hitachi).

Scanning electron microscopy. Samples were prefixed in $2 \%$ paraformaldehyde and $2 \%$ glutaraldehyde at $4{ }^{\circ} \mathrm{C}$ overnight. Postfixation was performed with $1 \%$ osmium tetroxide solution for 1 hour at room temperature. The samples were dehydrated in graded concentrations of ethanol $(30 \%, 50 \%, 70 \%, 90 \%, 95 \%$, and $100 \%)$ and dried with a critical point dryer (HCP-2, Hitachi) to remove residues of moisture. The samples were coated with platinum by cathodic spraying and then imaged with a scanning electron microscope (S-4700, Hitachi).

Immunocytochemistry labeling. For immunocytochemistry, the samples were treated as described previously (6). The primary antibodies used were antineurofilament (MilliporeSigma MAB5254, 1:500), anti-synaptic vesicle protein 2 (DSHB SV2, 1:50), anti-AChR $\gamma$ (antibodies-online, ABIN926968, 1:200), anti-AChRe (Santa Cruz Biotechnology Inc., sc-376826, 1:200), anti-MYH (MilliporeSigma A4.1025, 1:1000), anti-Islet1 (DSHB 40.2D6, 1:100), anti-HB9 (DSHB 81.5C10, 1:100), anti-ChAT (MilliporeSigma AB144P, 1:100), anti-S100 (Abcam ab14849, 1:300), anti-Tuj1 (Covance MRB435P, 1:1000), and synapsin1 (Millipore AB1543, 1:300). $\alpha$-Bungarotoxin, Alexa Fluor ${ }^{\mathrm{TM}} 647$ conjugate (Thermo Fisher Scientific) (Molecular Probes, B3545, $0.5 \mathrm{ug} / \mathrm{ml}$ ) was used to detect AChR. Samples were analyzed with confocal microscopy (Fluo View-1000, Olympus, or LSM-710, Zeiss).

Optogenetic activation. The plasmid containing a photoactivated domain was obtained from Addgene [pLenti-Synapsin-hChR2(H134R)-EYFP-WPRE, Addgene plasmid 20945]. To transduce the target gene into neuronal cells, the lentivirus transduction system (ViraPower HiPerform Lentiviral Expression Systems, Invitrogen) was used. NMJ cultures were treated with virus-containing medium at $37^{\circ} \mathrm{C}$ and $5 \% \mathrm{CO}_{2}$ for 48 hours. After viral infection, the medium was removed, and the Neurobasal medium was provided for target gene expression. The medium was refreshed every 3-4 days. EYFP expression was examined by confocal microscopy (LSM710, Zeiss) with proper optical settings. Photoactivation was performed at 488-nm laser wavelength. In addition to EYFP signals, bright-field images were captured simultaneously for muscle motion analysis. To inhibit muscle contraction, curare $(30 \mu \mathrm{g} / \mathrm{ml})$ (MilliporeSigma) was added to the culture medium. Pulsed blue light (450 nm) laser stimulation at $0.1,0.2,0.3$, and $0.5 \mathrm{~Hz}$ was also performed to evoke the neurons.

Preparation of SMN gene KD iPSCs (B $\left.7^{M Y O D}-S M N^{K D}\right)$. shRNA (TR301496, Origene) was used to knock down mRNA that encodes the SMN protein in iPSCs (B7 ${ }^{\mathrm{MYOD}}$ ). Then, NMJ differentiation was induced as above. We collected the cells 30 days later for further analysis.

Image quantification. For NMJ quantification, the fluorescence signals of neurons (neurofilaments + SV2) and AChRs were acquired in a 96-well plate. The number of ROIs depended on the lens magnification and was 16 or 25 ROIs/well. The areas of neurons and AChRs were measured with thresholds and then merged. The NMJ frequency was defined as merged area/neuron area (Supplemental Figure 2E). Image processing and quantification were performed by MetaMorph software (Molecular Devices) and In Cell Analyzer 6000.

Statistics. Statistics functions in Microsoft Excel 2013 were used for the statistical analyses. Data are expressed as mean \pm SEM. Statistical significance was determined using 2-tailed Student's $t$ test. In Figure $4 \mathrm{~K}$ and Supplemental Figure 4D, data were analyzed using 1-way ANOVA followed by Dunnett's test or Tukey's test (GraphPad Prism 7.02) $(P<0.05)$.

Study approval. The study plan for recombinant DNA research was approved by the Recombinant DNA Experiments Safety Committee of Kyoto University. The use of hESCs was approved by the Ministry of Education, Culture, Sports, Science and Technology of Japan.

\section{Author contributions}

MY, CYL, LTL, AI, SO, KN, HS, EM, TN, and MKS conceived the research. MY, CYL, and AI performed the experiments. MY, CYL, LTL, SO, and KN analyzed the data. MY, CYL, LTL, and MKS wrote the manuscript. 


\section{Acknowledgments}

The electron microscopy study was supported by Keiko Okamoto-Furuta and Haruyasu Kohda (Division of Electron Microscopic Study, Center for Anatomical Studies, Graduate School of Medicine, Kyoto University). We thank Yoko Nishinaka for supporting the flow cytometry analysis. We also thank Suguru Dowaki for the optogenetic analysis. The monoclonal antibody developed by HHMI/Columbia University was obtained from the Developmental Studies Hybridoma Bank, created by the National Institute Child Health and Human Development of the NIH and maintained at the Department of Biology, University of Iowa. We also thank Peter Karagiannis (CiRA, Kyoto University) for editing and proofreading the manuscript. This work was supported by funding support from Sony Imaging Products \& Solutions Inc. (to SO, KN, and EM); the Japan Society for the Promotion of Science KAKENHI, grant number 16H05352 (to MKS); iPS Cell Research Fund (to CYL and MKS); Mochida Memorial Foundation for Medical and Pharmaceutical Research (to MKS); Takeda Science Foundation (to MKS); and a grant from The Program for Intractable Diseases Research utilizing Disease-specific iPS cells, which was aided by the Japan Agency for Medical Research and Development (19bm0804005h0003 to HS, 17935400 to CYL and MKS, and 17935423 to MKS).

Address correspondence to: Megumu K. Saito, Department of Clinical Application, Center for iPS Cell Research and Application, Kyoto University, Kyoto, Japan, 606-8507. Phone: 81.75.366.7085/7089; Email: msaito@cira.kyoto-u.ac.jp. Or to: Michiko Yoshida, Department of Pediatrics, Kyoto Prefectural University of Medicine, Kyoto, Japan, 602-0841. Phone: 81.75.251.5571; Email: michi@koto.kpu-m.ac.jp.

1. Sanes JR, Lichtman JW. Development of the vertebrate neuromuscular junction. Annu Rev Neurosci. 1999;22:389-442.

2. Thomson SR, Wishart TM, Patani R, Chandran S, Gillingwater TH. Using induced pluripotent stem cells (iPSC) to model human neuromuscular connectivity: promise or reality? J Anat. 2012;220(2):122-130.

3. Witzemann V. Development of the neuromuscular junction. Cell Tissue Res. 2006;326(2):263-271.

4. Naumenko N, et al. Gender-specific mechanism of synaptic impairment and its prevention by GCSF in a mouse model of ALS Front Cell Neurosci. 2011;5:26.

5. Rodríguez Cruz PM, Palace J, Beeson D. Congenital myasthenic syndromes and the neuromuscular junction. Curr Opin Neurol. 2014;27(5):566-575.

6. Yoshida M, et al. Modeling the early phenotype at the neuromuscular junction of spinal muscular atrophy using patient-derived iPSCs. Stem Cell Reports. 2015;4(4):561-568.

7. Vilmont V, Cadot B, Ouanounou G, Gomes ER. A system for studying mechanisms of neuromuscular junction development and maintenance. Development. 2016;143(13):2464-2477.

8. Umbach JA, Adams KL, Gundersen CB, Novitch BG. Functional neuromuscular junctions formed by embryonic stem cell-derived motor neurons. PLoS One. 2012;7(5):e36049.

9. Steinbeck JA, et al. Functional connectivity under optogenetic control allows modeling of human neuromuscular disease. Cell Stem Cell. 2016;18(1):134-143.

10. Puttonen KA, Ruponen M, Naumenko N, Hovatta OH, Tavi P, Koistinaho J. Generation of functional neuromuscular junctions from human pluripotent stem cell lines. Front Cell Neurosci. 2015;9:473.

11. Demestre M, et al. Formation and characterisation of neuromuscular junctions between hiPSC derived motoneurons and myotubes. Stem Cell Res. 2015;15(2):328-336.

12. Tanaka A, et al. Efficient and reproducible myogenic differentiation from human iPS cells: prospects for modeling Miyoshi Myopathy in vitro. PLoS One. 2013;8(4):e61540.

13. Faravelli I, et al. Motor neuron derivation from human embryonic and induced pluripotent stem cells: experimental approaches and clinical perspectives. Stem Cell Res Ther. 2014;5(4):87.

14. Grady RM, Starr DA, Ackerman GL, Sanes JR, Han M. Syne proteins anchor muscle nuclei at the neuromuscular junction. Proc Natl Acad Sci U S A. 2005;102(12):4359-4364.

15. Oh Y, et al. Functional coupling with cardiac muscle promotes maturation of hPSC-derived sympathetic neurons. Cell Stem Cell. 2016;19(1):95-106.

16. Cohen-Cory S. The developing synapse: construction and modulation of synaptic structures and circuits. Science. 2002;298(5594):770-776.

17. Balice-Gordon RJ, Lichtman JW. In vivo observations of pre- and postsynaptic changes during the transition from multiple to single innervation at developing neuromuscular junctions. J Neurosci. 1993;13(2):834-855.

18. Lichtman JW, Colman H. Synapse elimination and indelible memory. Neuron. 2000;25(2):269-278.

19. Marques MJ, Conchello JA, Lichtman JW. From plaque to pretzel: fold formation and acetylcholine receptor loss at the developing neuromuscular junction. J Neurosci. 2000;20(10):3663-3675.

20. Jones RA, et al. Cellular and molecular anatomy of the human neuromuscular junction. Cell Rep. 2017;21(9):2348-2356.

21. Mishina M, et al. Molecular distinction between fetal and adult forms of muscle acetylcholine receptor. Nature. 1986;321(6068):406-411.

22. Walsh MK, Lichtman JW. In vivo time-lapse imaging of synaptic takeover associated with naturally occurring synapse elimination. Neuron. 2003;37(1):67-73.

23. Colman H, Nabekura J, Lichtman JW. Alterations in synaptic strength preceding axon withdrawal. Science. 
1997;275(5298):356-361.

24. Buffelli M, Busetto G, Bidoia C, Favero M, Cangiano A. Activity-dependent synaptic competition at mammalian neuromuscular junctions. News Physiol Sci. 2004;19:85-91.

25. Barik A, Zhang B, Sohal GS, Xiong WC, Mei L. Crosstalk between Agrin and Wnt signaling pathways in development of vertebrate neuromuscular junction. Dev Neurobiol. 2014;74(8):828-838.

26. Witzemann V, Chevessier F, Pacifici PG, Yampolsky P. The neuromuscular junction: selective remodeling of synaptic regulators at the nerve/muscle interface. Mech Dev. 2013;130(6-8):402-411.

27. Hayakawa T, et al. Noninvasive evaluation of contractile behavior of cardiomyocyte monolayers based on motion vector analysis. Tissue Eng Part C Methods. 2012;18(1):21-32.

28. Fletcher $P$, Forrester T. The effect of curare on the release of acetylcholine from mammalian motor nerve terminals and an estimate of quantum content. J Physiol (Lond). 1975;251(1):131-144.

29. Gorbe A, Becker DL, Dux L, Krenacs L, Krenacs T. In differentiating prefusion myoblasts connexin43 gap junction coupling is upregulated before myoblast alignment then reduced in post-mitotic cells. Histochem Cell Biol. 2006;125(6):705-716.

30. Araya R, et al. Expression of connexins during differentiation and regeneration of skeletal muscle: functional relevance of connexin43. J Cell Sci. 2005;118(pt 1):27-37.

31. Sáez JC, Cisterna BA, Vargas A, Cardozo CP. Regulation of pannexin and connexin channels and their functional role in skeletal muscles. Cell Mol Life Sci. 2015;72(15):2929-2935.

32. Ling KK, Gibbs RM, Feng Z, Ko CP. Severe neuromuscular denervation of clinically relevant muscles in a mouse model of spinal muscular atrophy. Hum Mol Genet. 2012;21(1):185-195.

33. Kariya S, et al. Reduced SMN protein impairs maturation of the neuromuscular junctions in mouse models of spinal muscular atrophy. Hum Mol Genet. 2008;17(16):2552-2569.

34. Wang ZB, Zhang X, Li XJ. Recapitulation of spinal motor neuron-specific disease phenotypes in a human cell model of spinal muscular atrophy. Cell Res. 2013;23(3):378-393.

35. Li DK, Tisdale S, Espinoza-Derout J, Saieva L, Lotti F, Pellizzoni L. A cell system for phenotypic screening of modifiers of SMN2 gene expression and function. PLoS One. 2013;8(8):e71965.

36. Ripolone M, et al. Impaired muscle mitochondrial biogenesis and myogenesis in spinal muscular atrophy. JAMA Neurol. 2015;72(6):666-675.

37. Guo X, Gonzalez M, Stancescu M, Vandenburgh HH, Hickman JJ. Neuromuscular junction formation between human stem cell-derived motoneurons and human skeletal muscle in a defined system. Biomaterials. 2011;32(36):9602-9611.

38. Patrón LA, August PR. Modeling neuromuscular junctions in vitro: a review of the current progress employing human induced pluripotent stem cells. AIMS Cell and Tissue Engineering. 2018;2(2):91-118.

39. Takemoto T, et al. Tbx6-dependent Sox2 regulation determines neural or mesodermal fate in axial stem cells. Nature. 2011;470(7334):394-398.

40. Turney SG, Lichtman JW. Reversing the outcome of synapse elimination at developing neuromuscular junctions in vivo: evidence for synaptic competition and its mechanism. PLoS Biol. 2012;10(6):e1001352.

41. Slater CR. The structure of human neuromuscular junctions: some unanswered molecular questions. Int J Mol Sci. 2017;18(10):E2183

42. Zhang F, Wang LP, Boyden ES, Deisseroth K. Channelrhodopsin-2 and optical control of excitable cells. Nat Methods. 2006;3(10):785-792.

43. Park GH, Kariya S, Monani UR. Spinal muscular atrophy: new and emerging insights from model mice. Curr Neurol Neurosci Rep. 2010;10(2):108-117.

44. Takahashi K, et al. Induction of pluripotent stem cells from adult human fibroblasts by defined factors. Cell. 2007;131(5):861-872.

45. Thomson JA, et al. Embryonic stem cell lines derived from human blastocysts. Science. 1998;282(5391):1145-1147.

46. Tennyson VM, Kremzner LT, Brzin M. Electron microscopic-cytochemical and biochemical studies of acetylcholinesterase activity in denervated muscle of rabbits. J Neuropathol Exp Neurol. 1977;36(2):245-275. 